\title{
Patient-Centered Research to Inform Patient-Centered Care for Adolescents and Young Adults (AYAs) With Cancer
}

\author{
Brad Zebrack, PhD, MSW, MPH
}

The 2 studies of young adult cancer survivors highlighted in this issue of Cancer are exemplars of a body of survivorship research that has emerged since Mullan's seminal article in the New England Journal of Medicine in 1985, which acknowledged a trajectory of survivorship that initiates at diagnosis and continues through extended phases of treatment and transitions into off-treatment survival. ${ }^{1}$ Both works evaluated patient-reported outcomes and thus provide insight into a patientcentered experience of cancer and its effect on the lives of adolescents and young adults (AYAs). Patient-centered perspectives on cancer survivorship are useful in informing the development of clinical care programs and research. They reflect the preferences, values, beliefs, and concerns of patients, which is knowledge necessary for equalizing the doctor-patient relationship, achieving shared decision-making, and perhaps contributing to a greater likelihood that patients will adopt provider instructions and adhere to and complete therapy. The studies by Salsman et al and Champion et al illuminate issues of particular relevance for an age-defined population of AYAs. Their findings are consistent with a small but emerging body of AYA survivorship literature and also stimulate thought related to methodological considerations for future AYA research.

Existing evidence suggests that patients' subjective experiences are better predictors of quality of life (QOL) outcomes relative to objective conditions. ${ }^{2-5}$ Few studies offer data to support the assertion that clinical characteristics such as cancer type or its associated severity or prognosis for survival explains variability in psychosocial outcomes. For example, a longitudinal study of 215 AYAs aged 15 to 39 years indicated that those with cancers that have $80 \%$ to $100 \%$ expected 5year survival rates (eg, testicular cancer, Hodgkin's disease) were just as likely to report psychological distress at 1-year postdiagnosis as were those with worse 5 -year survival rates. ${ }^{6}$ Thus, Salsman and colleagues had no a priori expectations for differences in outcomes by cancer type and were able to test a critical question related to time beyond end of therapy and its relationship to health-related QOL (HRQOL) in a large heterogeneous sample of AYAs. Given the relative rarity of cancer in teenagers and young adults, restricting studies of psychosocial outcomes to just 1 or 2 cancer types to control for presumed physiological effects related to cancer type unnecessarily burdens investigators. They require extended periods of time and/or large recruitment sources (multiple institutions or registries), and thus negatively affect the feasibility of conducting AYA trials with significant numbers of patients to test hypotheses.

Findings from the study by Champion et al raise important issues related to sex, sexuality, and the interdependence of relationships, emotional health, and physical functioning. As adolescents and young adults enter into intimate, emotional, and sexual relationships, perhaps for the very first time, they do not expect illness. The words so often spoken at the altar of marriage or commitment, "In sickness and in health, until death do us part," are rarely acknowledged by young people as a possibility as they consider other hopes and dreams for the future. Most AYAs, within the context of budding romances and new commitments, lack the skills necessary to navigate cancer and its challenges to relationships. Indeed, Champion et al reported impacts of cancer on mental health status, sexual functioning, physical functioning, and fatigue to be salient for AYAs, and significantly more so when compared to older patients with cancer. These factors are all interdependent and must be considered together when determining best approaches for supportive care; otherwise, interventions become piecemeal. For example, addressing sleep problems only by prescribing medication ignores attention to other known psychological or social correlates of sleep difficulties. Relying solely on pharmacological intervention for treating anxiety, depression, or adjustment disorders in patients with cancer, without considering psychotherapeutic options, flies in the face of evidence suggesting that psychotherapeutic and cognitive-behavioral interventions are

Corresponding author: Brad Zebrack, PhD, MSW, MPH, University of Michigan School of Social Work, 1080 S. University, Ann Arbor, MI 48109-1106; Fax: (734) 763-3372; zebrack@umich.edu

University of Michigan School of Social Work, Ann Arbor, Michigan

See original referenced articles on pages $2237-46$ and $2247-54$, this issue.

DOI: 10.1002/cncr.28734, Received: February 17, 2014; Revised: March 17, 2014; Accepted: March 28, 2014, Published online May 28, 2014 in Wiley Online Library (wileyonlinelibrary.com) 
efficacious. ${ }^{7,8}$ The work of Champion et al suggests the need for multifaceted, multipronged, multilevel research and interventions to improve QOL for AYA patients with cancer.

The studies by Salsman et al and Champion et al also suggest points of consideration regarding how we should be conducting AYA research. Specifically, their findings are salient to discussions of what the upper and lower age bounds could or should be to define an AYA population. Their findings also raise unique methodological issues related to cancer genetics and assessments of AYA family composition, gender identification, marital and relationship status, and sex and sexuality.

\section{AYA Age Range}

Of course, there are developmental differences within the 15- to 39-year age range, the convention for defining AYAs in the United States, ${ }^{9,10}$ and these developmental differences can confound study findings if age group subanalyses are not conducted. For example, we cannot draw conclusions about an age-bounded AYA population from the study by Champion et al, because they compared women under 45 years of age to older women. In contrast, Salsman et al did conduct subanalyses within the 15- to 39-year age cohort and found statistically significant differences in HRQOL between 30- to 39-year-olds and 25to 29-year-olds. The explanations for these differences are psychological and social in nature, and suggest that 30 - to 39-year-old "AYAs" may be more different from, than similar to, younger AYAs. Indeed, the developmental tasks, needs, and concerns for young people in their 30s are different from those of younger people in their teens and 20s. The continued inclusion of older AYAs aged 30 to 39 years in study samples may confound conclusions drawn about AYAs in general. The Salsman et al study findings appear to support a more narrow age range for an AYA population; however, they contrast with other studies in which no age differences were observed in emotional and mental health outcomes for similarly aged AYAs. ${ }^{6,11}$ Further research involving within-group as well as between-group comparisons is needed, as are more investigations of instrumentation and the possibility that use of standardized psychometric instruments not tested in an AYA population are producing spurious results.

\section{Genetics}

Although Champion and colleagues iterate that young women can and do get breast cancer, we also know that younger patients with breast cancer are more likely than older breast cancer patients to be diagnosed with metastatic disease and more likely to die from breast cancer. ${ }^{12}$ Moreover, the incidence of triple-negative breast cancer among younger women and particularly younger African American women, along with unique protein expression patterns and poorer survival, implicates varying gene-environment interactions with respect to age and race/ethnicity. ${ }^{13}$ An emerging field of epigenetics suggests that social and environmental stressors, including poverty and racial discrimination, have the potential to activate (or deactivate) genetic mechanisms and may place African American women in particular at increased risk for disease. Further research is needed to determine why younger women, and predominantly African American women, are diagnosed with this more deadly form of breast cancer, and to derive effective therapies.

\section{Family Composition, Gender, Marital/ Relationship Status, and Sex and Sexuality}

The authors of both studies identified limitations of their studies in terms of selection bias; however, there is an unmentioned aspect of selection bias that is of particular relevance to the AYA population, and it has to do with how investigators collect self-report data on family composition, gender, marital/relationship status, and sex and sexuality.

Today's AYAs are of a generation openly exposed to diverse family compositions. When surveying AYA patients about their parents and where they live, we must acknowledge that some young people are raised or live with just one parent or go back and forth between divorced or separated parents. Some AYAs have 2 mothers; some have 2 fathers. Some young people live temporarily with extended family members, sometimes to avoid parental abuse, neglect, alcoholism, or drug addiction. For some, living conditions are unstable to the point at which life is more about where you "stay at" and less so about "where you live." When asking AYAs where they live and with whom, survey response categories need to reflect the multiple and varied options that are relevant to them.

Similarly, male and female response categories for gender do not adequately reflect the realities of the human biological condition and may reflect investigators' own biases and ignorance in distinguishing biological sex and gender identification. When we include gender as a category in a survey, why? Are we suggesting that gender as biological sex assignment is relevant to our research question? Or is the respondent's gender identity—one's own internal, personal sense of being a man or a woman (or as someone outside of that gender binary) - more relevant? The answers to these questions guide us differently. If we think that one's gender identity is somehow relevant 
to the research, we must consider a universe of possible and mutually exclusive response options (male, female, transgender); otherwise, we increase the risk of bias by either excluding a portion of the population of interest, or else lessening the reliability of our findings by forcing persons to select a gender identity category that is not a true reflection of who they are.

The same holds true for questions (eg, marital status) that explicitly or implicitly assess sexual orientation: a person's enduring physical, romantic, and/or emotional attraction to another person (eg, straight, gay, lesbian, bisexual). Given that AYAs are at a time in their life when they are trying to make sense of themselves and their burgeoning sexuality, we enhance our own potential for deriving relevant knowledge about AYAs and the impact of cancer in their life if we use inclusive language and categories in our science. Given the existence of gay, lesbian, and bisexual AYAs diagnosed with cancer, we limit the rigor of our science by using language and response categories for collecting demographic data that excludes portions of the population and thus diminishes the probability of having representative samples. Questions related to marriage or relationship status must include scientifically valid response options that capture the realities of human sexual experience and minimize response bias.

As we proceed to advance an AYA research agenda, I offer one last comment having social and political implications. Although disparate survival and quality of life outcomes for AYAs have been reported, we must be careful about calling AYAs a "medically underserved group similar to racial and ethnic minorities." Like racial and ethnic minorities, AYAs have been subject to a lack of attention that is partially attributable to the organization of a health care service delivery system. Unlike racial and ethnic minority groups, however, the disparities in care and outcomes for AYAs are not rooted in hundreds of years of exposure to racism and discrimination. In considering submission of AYA research proposals to grantfunding mechanisms for "disparities research," we must be sensitive to a politics of research when comparing an AYA research agenda to one addressing disparate outcomes among minority groups (defined not only by race/ ethnicity but also by sexual orientation or gender), in which those disparities are rooted in institutionalized discrimination and social oppression. More importantly, however, all investigators involved in social and behavioral science and research need to recognize what unites us rather than what divides us. Together, we must focus our energies on collaboration and influencing the institutions responsible for allocating dollars for psychosocial and behavioral research, including disparities research however defined, and away from competing against one another for a diminishing piece of the research funding pie.

\section{CONFLICT OF INTEREST DISCLOSURES}

The authors made no disclosures.

\section{REFERENCES}

1. Mullan F. Seasons of survival: reflections of a physician with cancer. N Engl J Med. 1985;313:270-273.

2. Fleer J, Sleijfer D, Hoekstra H, et al. Objective and subjective predictors of cancer-related stress symptoms in testicular cancer survivors. Patient Educ Couns. 2006;64:142-150.

3. Täieb O, Moro MR, Baubet T, Revah-Lévy A, Flament MF. Posttraumatic stress symptoms after childhood cancer. Eur Child Adolesc Psychiatry. 2003;12:255-264.

4. Zebrack B, Landier W. The perceived impact of cancer on quality of life for post-treatment survivors of childhood cancer. Qual Life Res. 2011;20:1595-1608.

5. Cordova MJ, Andrykowski MA. Responses to cancer diagnosis and treatment: posttraumatic stress and posttraumatic growth. Semin Clin Neuropsychiatry. 2003;8:286-296.

6. Kwak M, Zebrack B, Meeske KA, et al. Trajectories of psychological distress in adolescent and young adult cancer patients: a one-year longitudinal study. J Clin Oncol. 2013;31:2160-2166.

7. Faller H, Schuler M, Richard M, et al. Effects of psycho-oncologic interventions on emotional distress and quality of life in adult patients with cancer: systematic review and meta-analysis. J Clin Oncol. 2013;31:782-793.

8. Hofmann SG, Asnaani A, Vonk IJ, Sawyer AT, Fang A. The efficacy of cognitive behavioral therapy: a review of meta-analyses. Cognit Ther Res. 2012;36:427-440.

9. Sender LS. A new journal to improve care for adolescent and young adult oncology patients and survivors. J Adolesc Young Adult Oncol. 2011;1:1-2.

10. Adolescent and Young Adult Oncology Progress Review Group. Closing the Gap: Research and Care Imperatives for Adolescents and Young Adults with Cancer, Report of the Adolescent and Young Adult Oncology Progress Review Group. NIH Publication No. 06-6067. Bethesda, MD: US Department of Health and Human Services, National Institutes of Health, National Cancer Institute; and the LiveSTRONG Young Adult Alliance. August 2006. http://planning. cancer.gov/library/AYAO_PRG_Report_2006_FINAL.pdf. Accessed April 18, 2014.

11. Smith AW, Bellizzi KM, Keegan TH, et al. Health-related quality of life in adolescent and young adult patients with cancer in the United States: the Adolescent and Young Adult Health Outcomes and Patient Experience Study. J Clin Oncol. 2013;31:2136-2145.

12. Johnson RH, Chien FL, Bleyer A. Incidence of breast cancer with distant involvement among women in the United States, 1976 to 2009. JAMA. 2013;309:800-805.

13. Lund MJ, Trivers KF, Porter PL, et al. Race and triple negative threats to breast cancer survival: a population-based study in Atlanta, GA. Breast Cancer Res Treat. 2009;113:357-370. 